PROCEEDINGS OF THE AMERICAN MATHEMATICAL SOCIETY

Volume 126, Number 4, April 1998, Pages 1237-1244

S 0002-9939(98)04316-0

\title{
THE CHERN CHARACTER FOR CLASSICAL MATRIX GROUPS
}

JAY A. WOOD

(Communicated by Thomas Goodwillie)

To S. S. Chern

\begin{abstract}
We give explicit formulas for representations of classical matrix groups whose Chern characters have lowest order terms equal to standard characteristic classes. For $\mathrm{SO}(2 r)$, the Euler class $e$ does not arise in this way, but $2^{r-1} e$ does arise in this way.
\end{abstract}

A complex representation $\rho$ of a compact Lie group $G$ gives rise to a complex vector bundle $\xi_{\rho}$ over the classifying space $B G$ of $G$. In this way one obtains a ring homomorphism $R(G) \rightarrow K(B G)$ from the complex representation ring of $G$ to the complex $K$-theory of $B G$. Composing with the Chern character yields a ring homomorphism,

$$
R(G) \rightarrow K(B G) \stackrel{\mathrm{ch}}{\longrightarrow} H^{* *}(B G ; \mathbb{Q}) .
$$

Our interest here is in determining which cohomology classes on $B G$ occur as the lowest order terms of some Chern character $\operatorname{ch}\left(\xi_{\rho}\right)$. We will consider the classical matrix groups $\mathrm{U}(n), \mathrm{SU}(n), \mathrm{Sp}(n)$, and $\mathrm{SO}(n)$, and we will produce explicit representations $\rho$ whose Chern characters $\operatorname{ch}\left(\xi_{\rho}\right)$ have lowest order terms equaling standard characteristic classes. In all cases considered here, the lowest order terms of the Chern characters $\operatorname{ch}\left(\xi_{\rho}\right)$ turn out to be integral classes. For $\mathrm{U}(n), \mathrm{SU}(n), \mathrm{Sp}(n)$, and $\mathrm{SO}(2 r+1)$, every homogeneous integral cohomology class modulo torsion on $B G$ arises as the lowest order term of some Chern character $\operatorname{ch}\left(\xi_{\rho}\right)$; for $\mathrm{SO}(2 r)$, the Euler class does not so arise, but $2^{r-1}$ times the Euler class does.

In the cases considered here where $B G$ has integral cohomology which is free of torsion, namely, $\mathrm{U}(n), \mathrm{SU}(n)$, and $\operatorname{Sp}(n)$, general results of Atiyah and Hirzebruch ([3], $\S 2.5$ and $\S 4.8)$ imply that the lowest order terms of any Chern character $\operatorname{ch}\left(\xi_{\rho}\right)$ are integral classes and that any homogeneous integral class arises in this way. Thus our only contribution in these cases is the explicit form of certain representations which give rise to standard characteristic classes.

For $\mathrm{SO}(n)$, whose classifying space has 2-torsion, the general results of Atiyah and Hirzebruch do not apply directly. It may be possible to obtain some general results in the presence of torsion by using the Atiyah-Hirzebruch spectral sequence together with integrality results such as those of Adams [1] and Maunder [7]; we have

Received by the editors October 1, 1996.

1991 Mathematics Subject Classification. Primary 55R40.

The author was partially supported by NSA grants MDA904-94-H-2025 and MDA904-96-10067, and by Purdue University Calumet Scholarly Research Awards. 
not pursued such general results here. Instead, we produce explicit representations which give rise to the Pontryagin classes and, when $n=2 r$, to $2^{r-1}$ times the Euler class. The explicit formulas we produce then allow us to show that the multiple $2^{r-1}$ is best possible.

We use, without comment, many facts from the representation and cohomology theories of these classical matrix groups. In particular, all computations really take place over a maximal torus $T$ via identifications $R(G) \cong R(T)^{W}$ and (valid for these groups) $H^{*}(B G ; \mathbb{Z}) /$ torsion $\cong H^{*}(B T ; \mathbb{Z})^{W}$. Relevant references are [5] and [6]. For notational convenience we will write $\operatorname{ch}(\rho)$ in place of $\operatorname{ch}\left(\xi_{\rho}\right)$.

We thank D. M. Davis, W. G. Dwyer, S. Gitler, R. J. Milgram, and S. Stolz for their helpful comments.

\section{1. $\mathrm{U}(n)$}

Let $\rho_{n}$ be the standard $n$-dimensional "birth-certificate" representation of $\mathrm{U}(n)$, and let $\Lambda^{i}=\Lambda^{i}\left(\rho_{n}\right)$ be the $i$ th exterior power of $\rho_{n}$. It is known that $R(\mathrm{U}(n))=$ $\mathbb{Z}\left[\Lambda^{1}, \Lambda^{2}, \ldots, \Lambda^{n}, \Lambda^{-n}\right]$. Also known is that $H^{*}(B \mathrm{U}(n) ; \mathbb{Z})=\mathbb{Z}\left[c_{1}, \ldots, c_{n}\right]$, where $c_{i} \in H^{2 i}(B \mathrm{U}(n) ; \mathbb{Z})$ is the $i$ th Chern class of $\xi_{\rho_{n}}$.

Upon restriction to a maximal torus $T$ of $\mathrm{U}(n)$, the representations $\Lambda^{i}$ split as follows:

$$
1+\Lambda^{1} t+\Lambda^{2} t^{2}+\cdots+\Lambda^{n} t^{n}=\prod_{i=1}^{n}\left(1+\alpha_{i} t\right),
$$

where $\alpha_{1}, \ldots, \alpha_{n}$ are the standard roots of $T$. It is well-known that $H^{*}(B T ; \mathbb{Z})=$ $\mathbb{Z}\left[x_{1}, \ldots, x_{n}\right]$, where $x_{1}, \ldots, x_{n}$ are classes of degree 2 with $x_{i}=c_{1}\left(\xi_{\alpha_{i}}\right)$. The work of Borel and Hirzebruch [4] (see [6], p. 282) tells us that the Chern character for bundles $\xi_{\rho}$ over $B T$ arises by replacing $\alpha_{i}$ by $e^{x_{i}}$. For example, we have our first proposition. (We shall use the notation нот to mean higher order terms.)

Proposition 1.1. On $\mathrm{U}(n)$, let $\rho=\Lambda^{n}-\Lambda^{n-1}+\Lambda^{n-2}-\cdots+(-1)^{n} \cdot 1$. Then $\operatorname{ch}(\rho)=c_{n}+$ HOT.

Proof. Setting $t=-1$ in (1.1), we have $\rho=\prod\left(\alpha_{i}-1\right)$. Then $\operatorname{ch}(\rho)=\prod\left(e^{x_{i}}-1\right)=$ $\prod\left(x_{i}+\right.$ нОт $)=c_{n}+$ нот.

Here we have made use of some standard facts concerning Chern classes. In the identification $H^{*}(B \mathrm{U}(n) ; \mathbb{Z}) \cong H^{*}(B T ; \mathbb{Z})^{W}, c_{i}$ equals the $i$ th elementary symmetric polynomial in $x_{1}, \ldots, x_{n}$. In particular, $c_{n}=\prod x_{i}$.

Next, we need to understand how the representations $\Lambda^{i}=\Lambda^{i}\left(\rho_{n}\right)$ of $\mathrm{U}(n)$ behave upon restriction to $\mathrm{U}(n-1)$. This amounts to setting $\alpha_{n}=1$ in (1.1). A short calculation leads to the next lemma.

Lemma 1.2. The restriction of the representation $\Lambda^{i}\left(\rho_{n}\right)$ to $\mathrm{U}(n-1)$ equals

$$
\Lambda^{i}\left(\rho_{n-1}\right)+\Lambda^{i-1}\left(\rho_{n-1}\right) .
$$

Theorem 1.3. For any $k, 1 \leq k \leq n$, let $d=n-k$, and set $\rho_{k, n}$ to be the following representation of $\mathrm{U}(n)$ :

$$
\begin{aligned}
\rho_{k, n} & =\sum_{j=0}^{k}(-1)^{j}\left(\begin{array}{c}
d+j \\
d
\end{array}\right) \Lambda^{k-j} \\
& =\Lambda^{k}-(d+1) \Lambda^{k-1}+\cdots .
\end{aligned}
$$


Then for any $m, k \leq m \leq n$, the restriction of $\rho_{k, n}$ to $\mathrm{U}(m)$ equals $\rho_{k, m}$. In particular, $\rho_{k, n}$ restricted to $\mathrm{U}(k)$ is $\rho_{k, k}=\Lambda^{k}-\Lambda^{k-1}+\Lambda^{k-2}-\cdots+(-1)^{k} \cdot 1$.

Proof. The general result will follow by induction once we know that $\rho_{k, n}$ restricts to $\rho_{k, n-1}$ on $\mathrm{U}(n-1)$. This is immediate from Lemma 1.2.

Corollary 1.4. Over $\mathrm{U}(n)$, we have $\operatorname{ch}\left(\rho_{k, n}\right)=c_{k}+$ HOT.

Proof. Restriction from $\mathrm{U}(n)$ to $\mathrm{U}(k)$ induces a projection

$$
H^{*}(B \mathrm{U}(n) ; \mathbb{Z}) \stackrel{i^{*}}{\longrightarrow} H^{*}(B \mathrm{U}(k) ; \mathbb{Z}),
$$

whose kernel is the ideal generated by $c_{k+1}, \ldots, c_{n}$. Since the Chern character is natural, we have

$$
i^{*} \operatorname{ch}\left(\rho_{k, n}\right)=\operatorname{ch}\left(\rho_{k, k}\right)=c_{k}+\text { нот, }
$$

by Proposition 1.1. But all the non-zero elements of ker $i^{*}$ have higher order than $c_{k}$, hence the result.

Remark 1.5. It turns out that $\rho_{k, n}=\gamma^{k}\left(\rho_{n}-n\right)$, where $\rho_{n}$ is the birth-certificate representation of $\mathrm{U}(n)$, and $\gamma^{k}$ is the $K$-theory operation due to Grothendieck (see [6], pp. 162-3). In factored form, $\rho_{k, n}$ is the $k$ th elementary symmetric polynomial in $\alpha_{1}-1, \ldots, \alpha_{n}-1$.

Corollary 1.6. Any non-zero homogeneous element of $H^{*}(B \mathrm{U}(n) ; \mathbb{Z})$ arises as the lowest order term of $\operatorname{ch}(\rho)$ for some $\rho \in R(\mathrm{U}(n))$.

Proof. Any homogeneous element $y$ of $H^{*}(B \mathrm{U}(n) ; \mathbb{Z})$ is expressible as a sum of monomials in the Chern classes $c_{k}$. Since the Chern character is a ring homomorphism, the corresponding sum of monomials in the $\rho_{k, n}$ will produce $y$ as its lowest order term.

\section{2. $\mathrm{SU}(n)$}

Since $\mathrm{SU}(n) \subset \mathrm{U}(n)$, the representations $\rho_{k, n}=\Lambda^{k}-(n-k+1) \Lambda^{k-1}+\cdots$ of $\mathrm{U}(n)$ restrict to $\mathrm{SU}(n)$ and have $\operatorname{ch}\left(\rho_{k, n}\right)=c_{k}+$ HOT. It turns out that we can do slightly better than this, achieving $c_{k}$ via a representation involving only $1, \Lambda^{1}, \ldots, \Lambda^{k-1}$.

Since $\Lambda^{n}=1$ over $\mathrm{SU}(n)$, we have $R(\mathrm{SU}(n))=\mathbb{Z}\left[\Lambda^{1}, \Lambda^{2}, \ldots, \Lambda^{n-1}\right]$. Also, $H^{*}(B \mathrm{SU}(n) ; \mathbb{Z})=\mathbb{Z}\left[c_{2}, \ldots, c_{n}\right]$, i.e., $c_{1}=0$.

Over $\mathrm{U}(n)$, the representation $\rho_{k, n}$ arose by finding representations over $\mathrm{U}(n)$ which would restrict to $\rho_{k, k}=\Lambda^{k}-\Lambda^{k-1}+\Lambda^{k-2}-\cdots+(-1)^{k} \cdot 1$ over $\mathrm{U}(k)$. For example, to find $\rho_{k, k+1}$, Lemma 1.2 requires $\rho_{k, k+1}$ to start with $\Lambda^{k}$. Restricting back to $\mathrm{U}(k)$, this $\Lambda^{k}$ will contribute $\Lambda^{k}+\Lambda^{k-1}$. Then $\rho_{k, k+1}$ needs $\Lambda^{k}-2 \Lambda^{k-1}$ to account for all the $\Lambda^{k-1}$ 's over $\mathrm{U}(k)$. This helps explain the origins of the $\rho_{k, n}$.

Over $\mathrm{SU}(n)$, we still have $\rho_{n, n}=\Lambda^{n}-\Lambda^{n-1}+\Lambda^{n-2}-\cdots+(-1)^{n} \cdot 1$, but now $\Lambda^{n}=1$. Thus we can write $\rho_{n, n}=-\Lambda^{n-1}+\Lambda^{n-2}-\cdots+(-1)^{n} \cdot 1+1$. As in the previous paragraph, we can lift $\rho_{n, n}$ to $\rho_{n, n+1}^{\prime}$, but now the formulas are different. 
Theorem 2.1. For any $k, 1 \leq k \leq n$, let $d=n-k$, and set $\rho_{k, n}^{\prime}$ to be the following representation of $\mathrm{SU}(n)$ :

$$
\begin{aligned}
\rho_{k, n}^{\prime} & =1+\sum_{j=0}^{k-1}(-1)^{j+1}\left(\begin{array}{c}
d+j \\
d
\end{array}\right) \Lambda^{k-1-j} \\
& =-\Lambda^{k-1}+(d+1) \Lambda^{k-2}-\cdots .
\end{aligned}
$$

Then for any $m, k \leq m \leq n$, the restriction of $\rho_{k, n}^{\prime}$ to $\mathrm{SU}(m)$ equals $\rho_{k, m}^{\prime}$. In particular, $\rho_{k, n}^{\prime}$ restricted to $\mathrm{SU}(k)$ is $\rho_{k, k}^{\prime}=\rho_{k, k}=-\Lambda^{k-1}+\Lambda^{k-2}-\cdots+(-1)^{k} \cdot 1+1$.

Corollary 2.2. Over $\mathrm{SU}(n)$, we have $\operatorname{ch}\left(\rho_{k, n}^{\prime}\right)=c_{k}+$ HOT. Any non-zero homogeneous element of $H^{*}(B \mathrm{SU}(n) ; \mathbb{Z})$ arises as the lowest order term of $\operatorname{ch}(\rho)$ for some $\rho \in R(\mathrm{SU}(n))$.

\section{3. $\operatorname{Sp}(n)$}

The birth-certificate representation of $\operatorname{Sp}(n)$ acts on $\mathbb{H}^{n}$. Restricting to the underlying complex vector space $\mathbb{C}^{2 n} \cong \mathbb{H}^{n}$, we obtain the standard complex representation $\rho_{2 n}$ of $\operatorname{Sp}(n)$. Let $\Lambda^{i}=\Lambda^{i}\left(\rho_{2 n}\right)$ be the $i$ th exterior power of $\rho_{2 n}, 1 \leq i \leq 2 n$. The representation $\rho_{2 n}$ preserves a non-degenerate symplectic form, and this implies isomorphisms $\Lambda^{i} \cong \Lambda^{2 n-i}$. While the $\Lambda^{i}$ are not irreducible, they do account for all of $R(\operatorname{Sp}(n))$ :

$$
R(\operatorname{Sp}(n))=\mathbb{Z}\left[\Lambda^{1}, \Lambda^{2}, \ldots, \Lambda^{n}\right] .
$$

Now $R(\operatorname{Sp}(n)) \cong R(T)^{W}$, with $T$ a maximal torus in $\operatorname{Sp}(n)$, and $R(T)=$ $\mathbb{Z}\left[\alpha_{1}, \alpha_{1}^{-1}, \ldots, \alpha_{n}, \alpha_{n}^{-1}\right]$. In this notation we have the splitting (over $T$ )

$$
1+\Lambda^{1} t+\cdots+\Lambda^{2 n} t^{2 n}=\prod_{i=1}^{n}\left(1+\alpha_{i} t\right)\left(1+\alpha_{i}^{-1} t\right) .
$$

Setting $t=-1$ and using $\Lambda^{i} \cong \Lambda^{2 n-i}$, we have

$$
\Lambda^{n}-2 \Lambda^{n-1}+2 \Lambda^{n-2}-\cdots+(-1)^{n} \cdot 2=\prod_{i=1}^{n}\left(\alpha_{i}+\alpha_{i}^{-1}-2\right) .
$$

$H^{*}(B \operatorname{Sp}(n) ; \mathbb{Z})$ is known to be torsion-free, and $H^{*}(B \operatorname{Sp}(n) ; \mathbb{Z}) \cong H^{*}(B T ; \mathbb{Z})^{W}$. If we write $H^{*}(B T ; \mathbb{Z}) \cong \mathbb{Z}\left[x_{1}, \ldots, x_{n}\right]$, with $\operatorname{deg} x_{i}=2$, then $H^{*}(B \operatorname{Sp}(n) ; \mathbb{Z}) \cong$ $\mathbb{Z}\left[q_{1}, q_{2}, \ldots, q_{n}\right]$, with $q_{i} \in H^{4 i}(B \operatorname{Sp}(n) ; \mathbb{Z})$. The element $q_{i}$ equals the $i$ th elementary symmetric polynomial in $x_{1}^{2}, \ldots, x_{n}^{2}$, and $q_{i}$ is called the $i$ th symplectic Pontryagin class.

Proposition 3.1. Over $\operatorname{Sp}(n)$, denote $\rho_{n, n}=\Lambda^{n}-2 \Lambda^{n-1}+2 \Lambda^{n-2}-\cdots+(-1)^{n} \cdot 2$. Then $\operatorname{ch}\left(\rho_{n, n}\right)=q_{n}+$ HOT.

Proof. From (3.2) we have $\operatorname{ch}\left(\rho_{n, n}\right)=\prod\left(e^{x_{i}}+e^{-x_{i}}-2\right)=\prod\left(x_{i}^{2}+\right.$ HOT $)=q_{n}+$ Hот.

Next we determine how the $\Lambda^{i}=\Lambda^{i}\left(\rho_{2 n}\right)$ behave under restriction to $\operatorname{Sp}(n-1)$. This amounts to setting $\alpha_{n}=\alpha_{n}^{-1}=1$ in (3.1). It then follows that $\Lambda^{i}\left(\rho_{2 n}\right)$ restricts over $\operatorname{Sp}(n-1)$ to $\Lambda^{i}\left(\rho_{2 n-2}\right)+2 \Lambda^{i-1}\left(\rho_{2 n-2}\right)+\Lambda^{i-2}\left(\rho_{2 n-2}\right)$. For what follows, set

$$
\left(\begin{array}{l}
n \\
k
\end{array}\right)^{\prime}=\left(\begin{array}{l}
n \\
k
\end{array}\right)+\left(\begin{array}{c}
n-1 \\
k
\end{array}\right) \text {. }
$$


Theorem 3.2. For any $k, 1 \leq k \leq n$, let $d=n-k$, and set $\rho_{k, n}$ to be the following representation of $\operatorname{Sp}(n)$ :

$$
\begin{aligned}
\rho_{k, n} & =\sum_{j=0}^{k}(-1)^{j+1}\left(\begin{array}{c}
2 d+j \\
2 d
\end{array}\right)^{\prime} \Lambda^{k-j} \\
& =\Lambda^{k}-(2 d+2) \Lambda^{k-1}+\cdots .
\end{aligned}
$$

Then for any $m, k \leq m \leq n$, the restriction of $\rho_{k, n}$ to $\operatorname{Sp}(m)$ equals $\rho_{k, m}$. In particular, $\rho_{k, n}$ restricted to $\operatorname{Sp}(k)$ is $\rho_{k, k}=\Lambda^{k}-2 \Lambda^{k-1}+2 \Lambda^{k-2}-\cdots+(-1)^{k} \cdot 2$.

Proof. This is a straight-forward verification.

Corollary 3.3. Over $\operatorname{Sp}(n)$, we have $\operatorname{ch}\left(\rho_{k, n}\right)=q_{k}+$ HOT. Any non-zero homogeneous element of $H^{*}(B \operatorname{Sp}(n) ; \mathbb{Z})$ arises as the lowest order term of $\operatorname{ch}(\rho)$ for some $\rho \in R(\operatorname{Sp}(n))$.

Proof. Since the restriction map

$$
H^{*}(B \operatorname{Sp}(n) ; \mathbb{Z})=\mathbb{Z}\left[q_{1}, \ldots, q_{n}\right] \rightarrow H^{*}(B \operatorname{Sp}(k) ; \mathbb{Z})=\mathbb{Z}\left[q_{1}, \ldots, q_{k}\right]
$$

has as kernel the ideal generated by $q_{k+1}, \ldots, q_{n}$, the same argument as in Corollary 1.4 applies.

\section{4. $\mathrm{SO}(n)$ : Pontryagin Classes}

The standard complex representation $\rho_{n}$ of $\mathrm{SO}(n)$ on $\mathbb{C}^{n}$ is the complexification of the birth-certificate representation of $\mathrm{SO}(n)$ on $\mathbb{R}^{n}$. Let $\Lambda^{i}=\Lambda^{i}\left(\rho_{n}\right)$ be the $i$ th exterior power of $\rho_{n}$. Because $\rho_{n}$ preserves the symmetric form coming from $\mathbb{R}^{n}$, we have isomorphisms $\Lambda^{i} \cong \Lambda^{n-i} ; \Lambda^{i}$ is irreducible for $i<n / 2$.

If $n=2 r+1$ is odd, this is all that happens:

$$
R(\mathrm{SO}(2 r+1))=\mathbb{Z}\left[\Lambda^{1}, \Lambda^{2}, \ldots, \Lambda^{r}\right]
$$

with

$$
1+\Lambda^{1} t+\cdots+\Lambda^{2 r+1} t^{2 r+1}=(1+t) \prod_{i=1}^{r}\left(1+\alpha_{i} t\right)\left(1+\alpha_{i}^{-1} t\right)
$$

being the splitting over a maximal torus $T$ (of rank $r$ ).

If $n=2 r$ is even, we have the splitting

$$
1+\Lambda^{1} t+\cdots+\Lambda^{2 r} t^{2 r}=\prod_{i=1}^{r}\left(1+\alpha_{i} t\right)\left(1+\alpha_{i}^{-1} t\right)
$$

over a maximal torus $T$. The middle dimensional $\Lambda^{r}=\Lambda^{r}\left(\rho_{2 r}\right)$ decomposes as $\Lambda^{r}=$ $\Lambda_{+}^{r}+\Lambda_{-}^{r}$ into self-dual and anti-self-dual parts. The representations $\Lambda^{1}, \ldots, \Lambda^{r-1}$, $\Lambda_{+}^{r}, \Lambda_{-}^{r}$ generate the representation ring $R(\mathrm{SO}(2 r))$, subject to a single relation (which we will not need to know explicitly).

Turning now to cohomology, $H^{*}(B \mathrm{SO}(n) ; \mathbb{Z})$ has 2 -torsion and

$$
H^{*}(B \mathrm{SO}(n) ; \mathbb{Z}) / \text { torsion } \cong H^{*}(B T ; \mathbb{Z})^{W} .
$$

Writing $H^{*}(B T ; \mathbb{Z})=\mathbb{Z}\left[x_{1}, \ldots, x_{r}\right]$, with $\operatorname{deg} x_{i}=2$, we have

$$
\begin{aligned}
& H^{*}(B \mathrm{SO}(2 r+1) ; \mathbb{Z}) / \text { torsion } \cong \mathbb{Z}\left[p_{1}, p_{2}, \ldots, p_{r}\right], \\
& H^{*}(B \mathrm{SO}(2 r) ; \mathbb{Z}) / \text { torsion } \cong \mathbb{Z}\left[p_{1}, p_{2}, \ldots, p_{r-1}, e\right],
\end{aligned}
$$


where the $i$ th Pontryagin class $p_{i} \in H^{4 i}(B \mathrm{SO}(n) ; \mathbb{Z}) /$ torsion is the $i$ th elementary symmetric polynomial in $x_{1}^{2}, \ldots, x_{r}^{2}$, and $e=x_{1} x_{2} \cdots x_{r}$ is the Euler class (when $n=2 r$ ). Of course, $e^{2}=p_{r}$. In this section we will show that every Pontryagin class arises as the lowest order term of some $\operatorname{ch}(\rho)$; the Euler class will be treated in the next section.

Let $\rho_{r, 2 r}=\Lambda^{r}-2 \Lambda^{r-1}+2 \Lambda^{r-2}-\cdots+(-1)^{r} \cdot 2$, a representation of $\operatorname{SO}(2 r)$. As usual, it arises by setting $t=-1$ in (4.2), so that

$$
\rho_{r, 2 r}=\prod_{i=1}^{r}\left(\alpha_{i}+\alpha_{i}^{-1}-2\right) .
$$

Just as for $\operatorname{Sp}(n)$, we have

Proposition 4.1. Over $\mathrm{SO}(2 r)$, let $\rho_{r, 2 r}=\Lambda^{r}-2 \Lambda^{r-1}+2 \Lambda^{r-2}-\cdots+(-1)^{r} \cdot 2$. Then $\operatorname{ch}\left(\rho_{r, 2 r}\right)=p_{r}+$ нот.

Using (4.1) and (4.2) we see that $\Lambda^{i}\left(\rho_{n+1}\right)$ of $\mathrm{SO}(n+1)$ restricts to $\Lambda^{i}\left(\rho_{n}\right)+$ $\Lambda^{i-1}\left(\rho_{n}\right)$ on $\mathrm{SO}(n)$. Remember the notation from (3.3).

Theorem 4.2. For any $k, 1 \leq k \leq n / 2$, let $d=n-2 k$, and set $\rho_{k, n}$ to be the following representation of $\mathrm{SO}(n)$ :

$$
\begin{aligned}
\rho_{k, n} & =\sum_{j=0}^{k}(-1)^{j}\left(\begin{array}{c}
d+j \\
d
\end{array}\right)^{\prime} \Lambda^{k-j} \\
& =\Lambda^{k}-(d+2) \Lambda^{k-1}+\cdots .
\end{aligned}
$$

Then for any $m, 2 k \leq m \leq n$, the restriction of $\rho_{k, n}$ to $\mathrm{SO}(m)$ equals $\rho_{k, m}$. In particular, $\rho_{k, n}$ restricted to $\mathrm{SO}(2 k)$ is $\rho_{k, 2 k}=\Lambda^{k}-2 \Lambda^{k-1}+2 \Lambda^{k-2}-\cdots+(-1)^{k} \cdot 2$.

Corollary 4.3. Over $\mathrm{SO}(n)$, we have $\operatorname{ch}\left(\rho_{k, n}\right)=p_{k}+$ HOT. Any non-zero homogeneous element of $H^{*}(B \mathrm{SO}(2 r+1) ; \mathbb{Z}) /$ torsion arises as the lowest order term of $\operatorname{ch}(\rho)$ for some $\rho \in R(\mathrm{SO}(2 r+1))$. The same is true for $\mathrm{SO}(2 r)$ provided the element is expressible entirely in terms of Pontryagin classes (including $p_{r}=e^{2}$; no odd powers of e are allowed).

\section{5. $\mathrm{SO}(2 r)$ : EULER CLASS}

Recall that on $\mathrm{SO}(2 r), \Lambda^{r}=\Lambda_{+}^{r}+\Lambda_{-}^{r}$. Since $\rho_{r, 2 r}=\Lambda^{r}-2 \Lambda^{r-1}+2 \Lambda^{r-2}-\cdots+$ $(-1)^{r} \cdot 2$ has $\operatorname{ch}\left(\rho_{r, 2 r}\right)=p_{r}+$ HOT, it makes sense to split $\rho_{r, 2 r}$ as $\rho_{r, 2 r}=\mu_{+}+\mu_{-}$ where

$$
\mu_{ \pm}=\Lambda_{ \pm}^{r}-\Lambda^{r-1}+\Lambda^{r-2}-\cdots+(-1)^{r} \cdot 1
$$

Theorem 5.1. Over $\mathrm{SO}(2 r)$ we have $\operatorname{ch}\left(\mu_{ \pm}\right)= \pm 2^{r-1} e+$ нот. No smaller multiple of the Euler class e arises as the lowest order term of $\operatorname{ch}(\rho)$ for any $\rho \in R(\mathrm{SO}(2 r))$.

Proof. We follow a computation of Atiyah and Hirzebruch, [2], §4.1.

The representations $\Lambda_{ \pm}^{r}$, when pulled back to $\operatorname{Spin}(2 r)$, are related to the spinor representations $\Delta^{ \pm}$by

$$
\begin{aligned}
& \Delta^{+} \otimes \Delta^{+}=\Lambda_{+}^{r}+\Lambda^{r-2}+\Lambda^{r-4}+\cdots, \\
& \Delta^{-} \otimes \Delta^{-}=\Lambda_{-}^{r}+\Lambda^{r-2}+\Lambda^{r-4}+\cdots .
\end{aligned}
$$

Observe that $\Lambda_{+}^{r}-\Lambda_{-}^{r}=\Delta^{+} \otimes \Delta^{+}-\Delta^{-} \otimes \Delta^{-}=\left(\Delta^{+}+\Delta^{-}\right)\left(\Delta^{+}-\Delta^{-}\right)$. 
Over a maximal torus for $\operatorname{Spin}(2 r)$ we have the splittings

$$
\Delta^{ \pm}=\sum_{\epsilon_{1} \cdots \epsilon_{r}= \pm 1} \alpha_{1}^{\epsilon_{1} / 2} \cdots \alpha_{r}^{\epsilon_{r} / 2}
$$

where $\epsilon_{i}= \pm 1$. Then

$$
\operatorname{ch}\left(\Delta^{ \pm}\right)=\sum_{\epsilon_{1} \cdots \epsilon_{r}= \pm 1} e^{1 / 2\left(\epsilon_{1} x_{1}+\cdots+\epsilon_{r} x_{r}\right)} .
$$

A straight-forward computation shows that

$$
\operatorname{ch}\left(\Delta^{+}+\Delta^{-}\right)=\prod_{i=1}^{r}\left(e^{x_{i} / 2}+e^{-x_{i} / 2}\right)
$$

and

$$
\operatorname{ch}\left(\Delta^{+}-\Delta^{-}\right)=\prod_{i=1}^{r}\left(e^{x_{i} / 2}-e^{-x_{i} / 2}\right) .
$$

Then $\operatorname{ch}\left(\Lambda_{+}^{r}-\Lambda_{-}^{r}\right)$ equals their product, i.e.,

$$
\operatorname{ch}\left(\Lambda_{+}^{r}-\Lambda_{-}^{r}\right)=\prod_{i=1}^{r}\left(e^{x_{i}}-e^{-x_{i}}\right)=2^{r} e+\text { нот. }
$$

Since $\mu_{+}+\mu_{-}=\rho_{r, 2 r}$ and $\mu_{+}-\mu_{-}=\Lambda_{+}^{r}-\Lambda_{-}^{r}$, we can solve for $\operatorname{ch}\left(\mu_{ \pm}\right)$:

$$
\operatorname{ch}\left(\mu_{ \pm}\right)=\frac{1}{2} \operatorname{ch}\left(\rho_{r, 2 r}\right) \pm \frac{1}{2} \operatorname{ch}\left(\Lambda_{+}^{r}-\Lambda_{-}^{r}\right) .
$$

Now, $\operatorname{ch}\left(\rho_{r, 2 r}\right)=p_{r}+$ нот, while $\operatorname{ch}\left(\Lambda_{+}^{r}-\Lambda_{-}^{r}\right)=2^{r} e+$ нот. But $p_{r}$ has degree $4 r$ while $e$ has degree $2 r$, so $\operatorname{ch}\left(\mu_{ \pm}\right)= \pm 2^{r-1} e+$ нот.

As for the sharpness of this result, we first observe that the inclusion

$$
R(\mathrm{SO}(2 r+1)) \rightarrow R(\mathrm{SO}(2 r))
$$

induced by restriction makes $R(\mathrm{SO}(2 r))$ a free module over $R(\mathrm{SO}(2 r+1))$, generated by 1 and $\Lambda_{+}^{r}$ (see [5], VI, (6.6)). The fact that $\mu_{+}$has the form

$$
\mu_{+}=\Lambda_{+}^{r}-\Lambda^{r-1}+\Lambda^{r-2}+\cdots+(-1)^{r} \cdot 1
$$

allows one to use 1 and $\mu_{+}$as the free module generators. In addition, the form of $\rho_{k, 2 r+1}=\Lambda^{k}-\cdots$ implies that

$$
R(\mathrm{SO}(2 r+1))=\mathbb{Z}\left[\Lambda^{1}, \ldots, \Lambda^{r}\right]=\mathbb{Z}\left[\rho_{1,2 r+1}, \ldots, \rho_{r, 2 r+1}\right] .
$$

The sharpness now follows from Corollary 4.3 and the first part of this theorem.

\section{REFERENCES}

[1] J. F. Adams, On Chern characters and the structure of the unitary group, Proc. Camb. Phil. Soc. 57 (1961), 189-199. MR 22:12525

[2] M. F. Atiyah and F. Hirzebruch, Quelques théorèmes de non-plongement pour les variétés différentiables, Bull. Soc. Math. France 87 (1959), 383-396. MR 22:5055

[3] _ Vector bundles and homogeneous spaces, Differential Geometry, Proc. Symp. Pure Math., vol. 3, Amer. Math. Soc., Providence, RI, 1963, pp. 7-38. MR 25:2617

[4] A. Borel and F. Hirzebruch, Characteristic classes and homogeneous spaces. I, Amer. J. Math. 80 (1958), 458-538. MR 21:1586

[5] Th. Bröcker and T. tom Dieck, Representations of compact Lie groups, Grad. Texts Math., vol. 98, Springer-Verlag, New York, Berlin, Heidelberg, and Tokyo, 1985. MR 86i:22023 
[6] D. Husemoller, Fibre bundles, 2nd ed., Grad. Texts Math., vol. 20, Springer-Verlag, New York, Heidelberg, and Berlin, 1975. MR 51:6805

[7] C. R. F. Maunder, Chern characters and higher order cohomology operations, Proc. Camb. Phil. Soc. 60 (1964), 751-764. MR 31:2722

Department of Mathematics, Computer Science \& Statistics, Purdue University Calumet, Hammond, Indiana 46323-2094

E-mail address: wood@calumet.purdue.edu 